\title{
Correction to: Tweaking Mesenchymal Stem/Progenitor Cell Immunomodulatory Properties with Viral Vectors Delivering Cytokines by Quaranta P, et al. Stem Cells Dev 2016;25(18):1321-1341 DOI:10.1089/scd.2016.0145
} THE AUTHORS OF THIS ARTICLE entitled Tweaking Mesenchymal Stem/Progenitor Cell Immunomodulatory
Properties with Viral Vectors Delivering Cytokines, published in Stem Cells Dev 2016;25;18:1321-1341 wish to correct
an error.

In the original Comprehensive Review, page 1321, right column, the text read as follows:

"Currently, naive hMSCs are employed in a large number of clinical trials successfully and, as a result, the first cell-based therapy based on allogeneic MSC-derived drugs has been approved by the Food and Drug Administration (FDA) [7].",

It has been revised to read as:

"Currently, naïve hMSCs are successfully employed in a large number of clinical trials [7] and, as a result, the first cell-based therapy based on allogenic MSCs-derived drug has been approved by the Health Canada."

In the original Comprehensive Review, page 1326, left column, the text read as follows:

"As a result, the drug Prochymal (Osiris Therapeutics, Inc., Columbia, MD), based on allogeneic MSCs derived from the BM of adult donors, was approved by the FDA to treat acute GVHD."

It has been revised to read as:

"As a result, the drug ProchymalTM (Osiris Therapeutics Inc., Columbia, MD), based on allogenic MSCs derived from the BM of adult donors, was approved by the Health Canada to treat acute GVHD."

The online version of this article has been revised to reflect this correction.

The authors sincerely regret this error. 